\title{
Molecular regulation of normal and leukaemic human haemataopoeitic stem cells
}

\author{
Tariq Enver ${ }^{1}$ \\ ${ }^{I}$ MRC Molecular Haematology Unit, Weatherall Institute of Molecular Medicine, John Radcliffe Hospital, University of Oxford, \\ $U K$
}

Despite being amongst the best of characterized of somatic stem cells, the molecular mechanisms underlying the regulation of blood stem cell fates continue to attract significant attention. Much of what is known has been gleaned from studies of mouse HSC and while many mechanisms will be conserved between mouse and humans through evolution it is likely that many will not and consequently clinical application requires prior study of human cells. Such studies are made more urgent by well known differences in the growth and transformation properties of human and mouse cells. We have therefore been focusing on these issues in human cord bloodderived haematopoietic stem cells (cord blood) and have identified novel regulators of human stem cell selfrenewal (Nov/CCN3) and lineage commitment (MLLT3/AF9). In addition we have explored the nature of prenatally derived stem cells which may act as targets for initiating molecular events in childhood ALL, the commonest cancer of children. We have identified a candidate stem cell which initiates and maintains the peleukaemic phase of the disease and through xenograft modeling determined how the $t(12 ; 21)$-generated, TELAML fusion gene corrupts the programme of a pre-natallydrived stem/progenitor cell to initiate leukaemogenesis.

Key words: HSC, cord blood, leukaemia, transcription factors, lineage commitment

Cell Research (2008) 18:s92. doi: 10.1038/cr.2008.182; published online 4 August 2008

Correspondence: Tariq Enver

E-mail: tenver@gwmail.jr2.ox.ac.uk

Tariq Enver PhD is currently Professor of Stem Cell biology at the University of Oxford and Director of Stem Cell Research at the Medical Research Council's Molecular Haematology Unit in the Weatherall Institute for Molecular Medicine on the John Radcliffe Hospital Site. His research career has been principally concerned with understanding the mechanisms by which tissue and developmental stage specific gene expression is achieved and regulated with early work focusing on the regulation of the -globin gene clusters. Later work focussed on describing the molecular groundstate of stem cells leading to the development of the multi-lineage priming hypothesis which provides both a mechanistic framework for for lineagespecification and explanation for the mixed-lineage phenotypes observed in many leukaemic cells. His current work deploys post-genomic technologies and mathematical modelling approaches to gain further insight into how blood stem cells are configured in molecular terms, the nature of the pathways involved in their cell fate decisions, and how these are corrupted by chimaeric transcription factors associated with human leukaemia. Studies addressing the latter focus primarily on the TEL-AML1 fusion gene which is the most common genetic abnormality in childhood cancer. Please see his website for more details. 